\title{
Brainwear: Towards Multi-modal Garment Integrated EEG
}

\author{
Juan F. Vargas \\ DFKI GmbH and TU Kaiserslautern \\ Germany \\ juan.vargas@dfki.de \\ Hymalai Bello \\ DFKI GmbH and TU Kaiserslautern \\ Germany \\ hymalai.bello@dfki.de
}
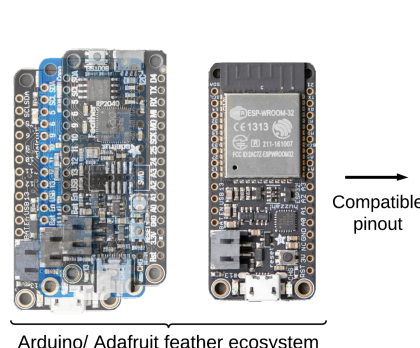

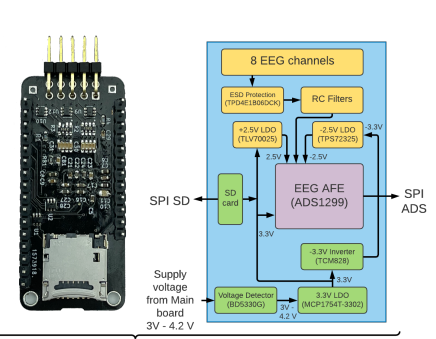

Brainwear as a Feather wing
Bo Zhou

DFKI GmbH and TU Kaiserslautern

Germany

bo.zhou@dfki.de

Paul Lukowicz

DFKI GmbH and TU Kaiserslautern

Germany

paul.lukowicz@dfki.de

Figure 1: Left: Brainwear is compatible with the Adafruit Feather footprint. Right: aesthetical wearable for HAR and multi-modal probe.

\begin{abstract}
We aim to facilitate broad use of EEG sensing in multi-modal smart garments by developing an open-source EEG sensing module with the state-of-the-art analog front-end that is pin/protocol-compatible with popular ecosystems in the wearable and DIY community. The EEG functionality is validated with the neuroscience standard nback memory load task. We also demonstrate the seamless integration of EEG electrodes with low-frequency Force Sensitive Resistors (FSR) and high-frequency piezoelectric sensors within a single probe. Finally, we show the embedding of the entire setup in a textile baseball cap. We also present how signals from the different modalities complement each other under situations such as motion artifacts and different activities from an unobtrusive head-worn garment. The system is available to the community through a public GitHub repository.
\end{abstract}

\section{CCS CONCEPTS}

- Computer systems organization $\rightarrow$ Embedded systems.

\section{KEYWORDS}

Wearable EEG; Brain-Computer-Interface; Open-source toolkit

Permission to make digital or hard copies of all or part of this work for personal or classroom use is granted without fee provided that copies are not made or distributed for profit or commercial advantage and that copies bear this notice and the full citation on the first page. Copyrights for components of this work owned by others than ACM must be honored. Abstracting with credit is permitted. To copy otherwise, or republish, to post on servers or to redistribute to lists, requires prior specific permission and/or a fee. Request permissions from permissions@acm.org.

ISWC '21, September 21-26, 2021, Virtual, USA

(C) 2021 Association for Computing Machinery.

ACM ISBN 978-1-4503-8462-9/21/09 . \$ \$15.00

https://doi.org/10.1145/3460421.3478814

\section{ACM Reference Format:}

Juan F. Vargas, Bo Zhou, Hymalai Bello, and Paul Lukowicz. 2021. Brainwear: Towards Multi-modal Garment Integrated EEG. In 2021 International Symposium on Wearable Computers (ISWC '21), September 21-26, 2021, Virtual, USA. ACM, New York, NY, USA, 6 pages. https://doi.org/10.1145/3460421.3478814

\section{INTRODUCTION}

Sensing brain activity, including (but not limited to) Brain-Computer Interfaces (BCI), is increasingly becoming an active research topic in wearable computing. There are several initial consumer-grade applications as explored in [23]. Most work on brain activity monitoring uses non-invasive EEG for its high temporal resolution, low cost, and portability capabilities. [30]. However, most current commercial wearable EEG devices have not gained high adoption due to issues like comfort and social acceptance, especially for long-term use. Most EEG work is constrained to laboratory environments in which the user's movement is restricted due to high sensitivity and motion artifacts, thus limiting the research questions that such studies can address [23]. Hence, research and future consumer applications towards the "Transparent EEG" concept[4] require long-term solutions that are at the same time aesthetically appealing, easy-to-use, motion/noise-tolerant. For the latter, in particular, close integration with additional sensors is a promising approach. Finally, broadly accessible platforms that can be easily used and extended by the community are needed [20]. While addressing such usability and multi-modal issues is the forte of the collective wearable community, the current barrier encapsulating EEG technologies has so far discouraged highly needed advancements.

In our summary of neuroscience studies regarding human activity recognition (HAR) in Section 2, EEG proves to be a potent sensing modality. Yet, the adaptation of EEG in wearable HAR studies has so far been limited due to the reasons explained above. Thus, we developed an open-source EEG module that is hardware compatible with the popular Adafruit Feather family based on the Arduino 
ecosystem. We have also demonstrated how EEG electrodes can be seamlessly stack-integrated with FSR and piezoelectric sensors within a single probe. This design initiative aims to bring the EEG modality more accessible to the wearable computing community to promote future studies with EEG-integrated garments combining other sensing modalities.

To bridge the gap between wearable BCI and HAR, our contributions are:

(1) We developed a compact, easy-to-use Arduino-based EEG module, Brainwear, which we provide as a complete opensource toolkit to the community ${ }^{1}$.

(2) We implemented a proof of concept application of the system in a normal baseball cap.

(3) The EEG performance in the cap prototype was evaluated on a standard neuroscience benchmark (n-back).

(4) We showed that additional sensing modalities, specifically FSR (pressure sensing) and piezoelectric, can be integrated within each single sensor probe in the cap.

(5) On a set of example signals, we demonstrate and discuss the benefit of combining EEG with the additional sensors in a single device.

\section{BACKGROUND AND STATE OF THE ART}

In the wearable HAR discipline, EEG has already shown great potential [29]. As summarized in Table 1, EEG can provide information on many psychological and cognitive aspects where physiological sensors usually struggle. However, none of these studies has seamlessly integrated EEG with other physiological modalities in a single embedded system - the EEG signal is always provided by specialized hardware with obvious barriers of garment integration.

Ad hoc EEG systems or components have been created in various studies. For example, [7] developed novel 3D-printed electrodes that are located into an e-Textile headband. [20] combined the around-the-ear electrodes (cEEGrigs) proposed by [4] and the OpenBCI platform to create a ready-to-wear device to recognize mental workload as well as other BCI tasks.

Recent commercial mobile EEG devices, such as Mindwave (Neurosky), Muse (InteraXon), and EPOC (Emotiv) [30], have reduced the design overhead of ad hoc prototypes for BCI studies[20]. Besides the usual discomfort and fitting issues, such rigid devices fall short as the proprietary system makes it impossible for the wearable community to integrate them into multi-modal, ergonomic, and aesthetic designs.

Open-source toolkits such as OpenBCI provide hardware and software suites integrating EEG Analog-Front-End (AFE) in a complete embedded system, compatibility with several types of electrodes, and flexibility to measure brain signals at any location on the scalp. Yet, OpenBCI has only seen limited adaptation among wearable researchers and smart garment designers outside the BCI community due to the low compatibility of the processor and software layers together with relatively high entry barrier and overhead. Additionally, its full-stack solution also has difficulty keeping up and integrating with the latest advancements in sectors like microprocessors, communication, and software.

\footnotetext{
${ }^{1}$ https://github.com/jufvargasco/Brainwear2.0
}

The true wearable EEG enabler is the latest generations of chips integrating EEG-level AFE, such as the ADS1299 (Texas Instruments) that integrates the entire 8-channel AFE in a compact package, replacing traditional cumbersome circuit assembly. ${ }^{2} \mathrm{Sev}^{-}$ eral studies [11, 27, 30] have concluded that the ADS1299 reference design provides data on par with medical-grade systems.

In neuroscience, discarding the signals contaminated by motion artifacts is a common practice [5,17], as muscular, ocular, and cardiac activities are coupled with electrical signals surpassing the surface EEG magnitude. However, these signals can also be interesting for HAR purposes. Facial muscular activities, for example, are shown to be relevant for emotional control [2], motor planning [28], emotional expressions [36], reading activities [21] and snacking moments [29, 37]. With the latest dry and textile electrode breakthroughs such as $[1,10,32]$, the combination of multiple sensors to detect muscle movements in parallel with EEG is the apparent way forward [13, 29].

\section{COMMUNITY-ORIENTED HARDWARE}

The Brainwear EEG module's major design considerations are simplicity and compatibility. Simplicity by packaging all and only the necessary components for EEG sensing so that designers can use it as a plug-and-play module; and compatibility with the open and popular standards to not be locked-in by legacy technology. We positioned Brainwear as a plug-and-play part of the broader wearable system options so that EEG-enabled devices can easily benefit from the latest advancements such as faster processors, more efficient wireless communication, edge computing acceleration, and beyond.

As shown in Fig. 1, enabled by ADS1299[15], the Brainwear module handles up to 8 EEG channels and output the digital data by the SPI bus. More channels can be achieved by either daisy-chaining on the same SPI bus or adding modules on more SPI buses, depending on the host microprocessor's capability. Brainwear is designed to be footprint and pin-compatible with the Adafruit Feather family, which contains over 100 different modules with the same board footprint. Feathers are programmed with the Arduino software environment, which means a wide selection of microcontrollers can drive the Brainwear module with the same code. Even if designers choose microcontroller modules outside the Feather family, Brainwear can still be easily integrated by plugging into the SPI bus with minimal code modification, including OpenBCI.

The design, including technical specifications and board manufacturing files, together with the Arduino C library and examples, are all available on GitHub, so the entire wearable community can easily reproduce and integrate into their designs and studies.

\section{PROTOTYPE VALIDATION}

We first validated our approach with a soft EEG cap prototype ${ }^{3}$ and the standard benchmark N-back test, through a study based on the common approach in neuroscience $[8,16,26]$.

\footnotetext{
${ }^{2}$ OpenBCI Cyton and [7] both use ADS1299 with the chipmaker's reference design ${ }^{3}$ To validate the soft EEG cap with a cognitive study while performing in parallel the printed circuit board design process. In this prototype, we used Cyton (OpenBCI), which uses the same reference design as the ADS1299. The later signal level comparison showed no distinguishable difference between Brainwear and Cyton
} 
Table 1: Summary of recent studies using mobile EEG for psychological studies and Human Activity Recognition

\begin{tabular}{|c|c|c|c|c|c|}
\hline Application & Activities & Sensors and biosignals & Classifier & Accuracy & \\
\hline \multirow{4}{*}{ Emotions } & valence, arousal, happiness, fear, and excitement & $\begin{array}{l}\text { OpenBCI (EEG) } \\
\text { Empatica4 (EDA, skin temp, PPG) }\end{array}$ & k-means clustering & $\begin{array}{l}67 \% \text { valence } \\
70 \% \text { arousal }\end{array}$ & {$[22]$} \\
\hline & valence, arousal, and dominance & $\begin{array}{l}\text { Emotiv EPOC (EEG) } \\
\text { SHIMMER (ECG) }\end{array}$ & SVM & 62 average & [18] \\
\hline & Anxiety ( 3 different levels) & $\begin{array}{l}\text { MindWave Mobile (EEG) } \\
\text { PPG-fitted glasses (PPG) }\end{array}$ & KNN & $62.5 \%$ & [35] \\
\hline & Stress & $\begin{array}{l}\text { MindWave Mobile (EEG) } \\
\text { SHIMMER (EDA) } \\
\text { BioHarness } 3 \text { (ECG) }\end{array}$ & SVM & $86 \%$ & [3] \\
\hline \multirow{2}{*}{ Cognition } & Mental workload (0,1,2,3 - back) & Emotiv EPOC (EEG) & SVM & $81 \%$ ( 0 vs $1 / 2 / 3$-back) & [34] \\
\hline & Drowsiness and Fresh state & Muse (EEG, IMU) & SVM & $92 \%$ & [24] \\
\hline $\begin{array}{l}\text { Emotion/ } \\
\text { cognition }\end{array}$ & Success in a learning task & $\begin{array}{l}\text { Emotiv EPOC (EEG) } \\
\text { Tobii Tx300 (Eye movement) } \\
\text { FaceReader } 6.0 \text { (Expression) }\end{array}$ & Logistic regression & $66 \%$ & {$[12]$} \\
\hline Motion & $\begin{array}{l}\text { Hand movement speed and position } \\
\text { (fast-right, fast-left, slow-right, slow left) }\end{array}$ & $\begin{array}{l}\text { Emotiv EPOC (EEG) } \\
\text { V120 Duo (Limb motion) }\end{array}$ & $\begin{array}{l}\text { LDA } \\
\text { SVA }\end{array}$ & $\begin{array}{l}73.72 \% \text { Only speed } \\
69 \% \text { left/right movement }\end{array}$ & [28] \\
\hline Daily activities & Reading, speaking, watching TV & Muse (EEG) & FCEA framework & $94.60 \%$ & [29] \\
\hline Dancing & $\begin{array}{l}\text { Neutral, think, and do } \\
\text { Laban efforts (17 classes) }\end{array}$ & $\begin{array}{l}\text { BrainAmpdDC (EEG) } \\
\text { OPAL (Body kinematics) }\end{array}$ & $\begin{array}{l}\text { LFDA + } \\
\text { GMM }\end{array}$ & $\begin{array}{l}59.4 \% 3 \text { classes } \\
88.2 \% 17 \text { classes }\end{array}$ & [9] \\
\hline $\begin{array}{l}\text { Cognitive }+ \\
\text { motor tasks }\end{array}$ & $\begin{array}{l}\text { Relax, visual task, auditive task }+7 \text { motor tasks (organiz- } \\
\text { ing books, standing up, salute motions, clapping, etc.) }\end{array}$ & $\begin{array}{l}\text { Enobio (EEG) } \\
\text { Rokoko (IMU) }\end{array}$ & $\begin{array}{l}\text { Random Forest } \\
\text { Gradient boost }\end{array}$ & $96 \%$ & [13] \\
\hline
\end{tabular}
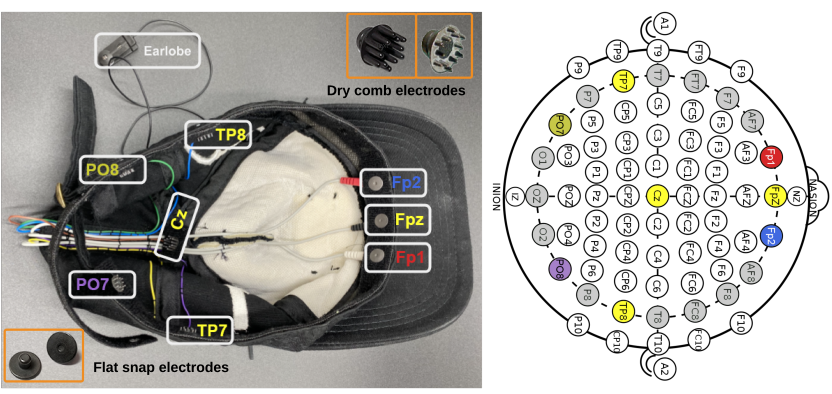

Figure 2: Soft EEG cap and electrode placement.

\subsection{Validation Method}

Based on the analysis performed in [25] about the regions involved in memory workload and the 10-20 system [19], we located the dry electrodes at approximately evenly spaced locations at the prefrontal cortex (FP1, FP2, and FPz), the premotor cortex $(\mathrm{Cz})$, and intersections between the temporal and occipital lobes with the parietal lobe (TP8, TP7, PO8, and PO7). Flat electrodes for hairless regions and comb electrodes of 2 or $5 \mathrm{~mm}$ long prongs for hairy regions are used depending on the participant's hairstyle. ${ }^{4}$ The integrated cap and the electrode placement is shown in Fig. 2.

Five healthy participants with university education (2 females, 3 males) between 23 and 30 years of age (mean age 26) took part in the experiment. All subjects were right-handed, had a normal or corrected-to-normal vision, and were naive to the nature of the experiment.

The experiment goal is to classify five different levels of memory workload: resting state with eyes closed (EC) and eyes opened (EO), and three states with increasing memory load: 1-, 2-, and 3-back. The single n-back task (Brain Workshop) with visual stimuli was used to induce cognitive load and benchmark working memory.

${ }^{4}$ Such dry electrodes are available from Florida Research Instruments Inc, Unicorn-BI, Neurospec, etc.
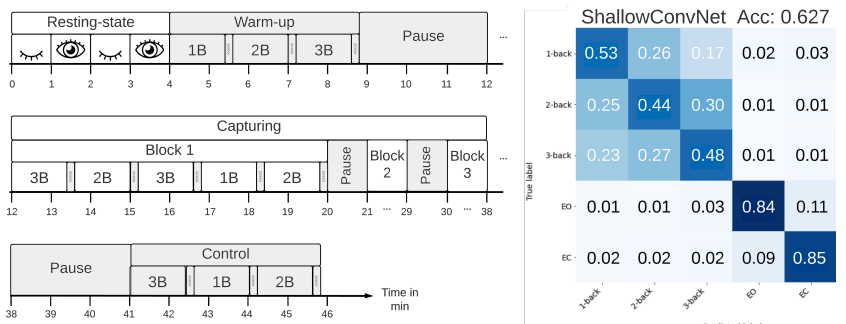

Figure 3: Experiment procedure and result.

Fig. 3 shows the time course of the experimental procedure, containing five pseudo-randomized iterations of the three n-back levels. Each participant finished two sessions with a total of 4 minutes for each resting state and 21 minutes for each $n$-back level. The experiment was presented on a 24-inch monitor located at about $50 \mathrm{~cm}$ distance from participants and took place in one of the experimental rooms of the DFKI.

The signal processing and machine learning pipeline are based on the mature consensus and state-of-the-art in neuroscience [34]. The 8-channel $250 \mathrm{~Hz}$ raw signal was upsampled to $256 \mathrm{~Hz}$ by applying an FIR anti-aliasing filter. Then, the DC offset was removed by a high-pass filter with a cutoff frequency at $0.5 \mathrm{~Hz}$. Moreover, a notch filter was used to remove the line noise at $50 \mathrm{~Hz}$. The processed data was segmented into non-overlapping $2 \mathrm{~s}$ windows. For $\mathrm{n}$-back states, only the windows in the Capturing Stage (Figure 3) are used as the participants are properly engaged.

We employed a CNN-based deep neural network model commonly used in EEG signal classification: the Shallow ConvNet [31]. ${ }^{5}$ Leave-one-session-out cross-validation was performed. Weightassignment was used to tackle the data imbalance between idle states and engaged states.

${ }^{5}$ The model was taken from the Army Research Laboratory (ARL) EEGModels project https://github.com/vlawhern/arl-eegmodels, adapted to our data. 


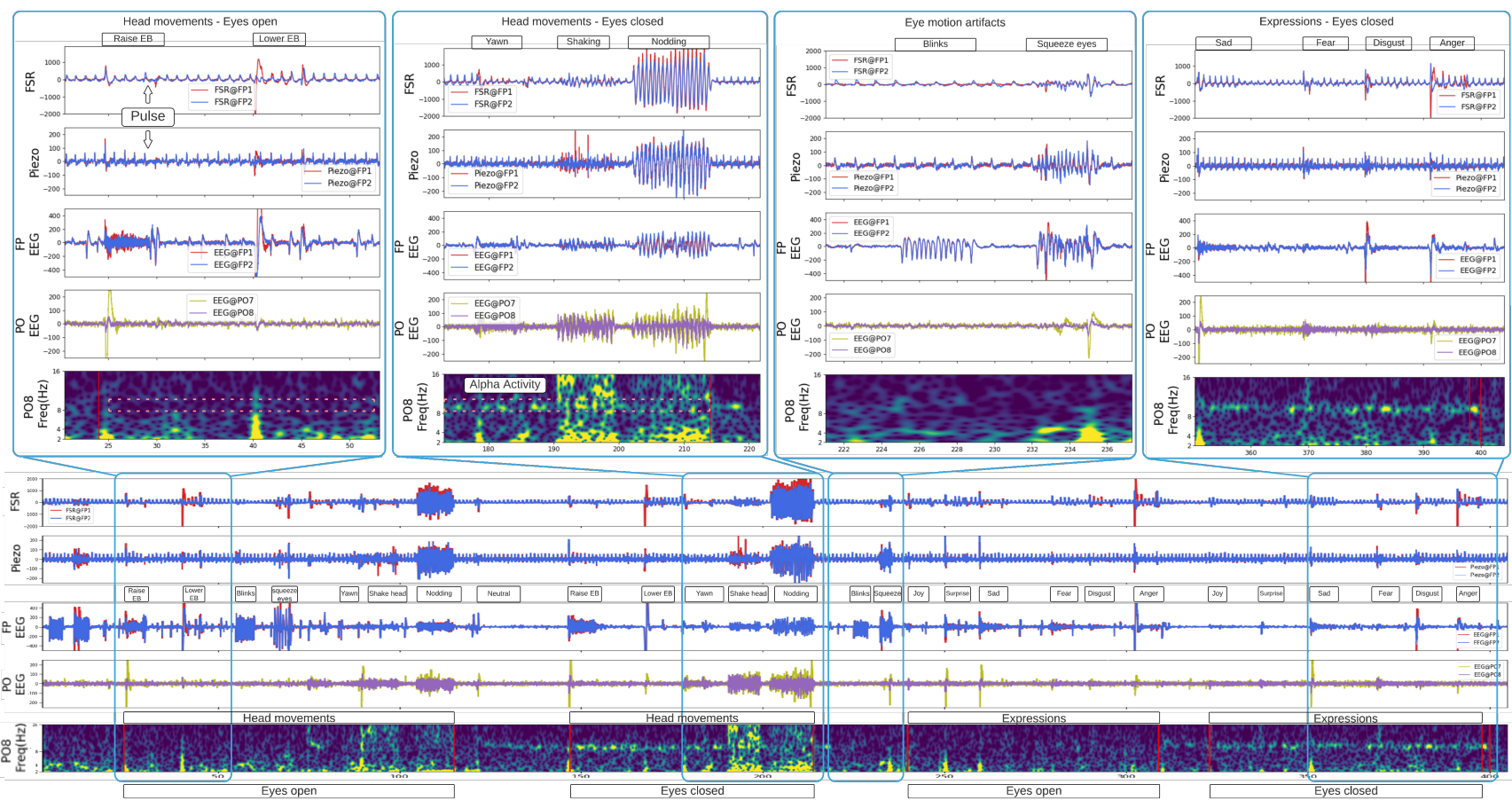

Figure 4: Person wearing the multi-modality cap while performing head movements and facial expressions with eyes closed and eyes open.

Table 2: Comparison with the neuroscience related work

\begin{tabular}{|c|c|c|c|c|}
\hline Studies & Device & $\begin{array}{l}\text { Memory } \\
\text { workload } \\
\text { levels }\end{array}$ & $\begin{array}{l}\text { Classification } \\
\text { method }\end{array}$ & $\begin{array}{l}\text { Accuracy } \\
\text { 2s window }\end{array}$ \\
\hline $\begin{array}{l}\text { Grimes } \\
\text { et al. [14] }\end{array}$ & $\begin{array}{l}\text { Biosemi } \\
\text { activetwo } \\
(32-\mathrm{ch})\end{array}$ & $\begin{array}{l}0 \text { to } 3 \text {-back } \\
\text { (letter, image, } \\
\text { spatial tasks) }\end{array}$ & $\begin{array}{l}\text { Naïve Bayes } \\
\text { (2-classes, } \\
\text { 4-classes) }\end{array}$ & $\begin{array}{l}30 \% \\
\text { (4-classes) }\end{array}$ \\
\hline $\begin{array}{l}\text { Brouwer } \\
\text { et al. [6] }\end{array}$ & $\begin{array}{l}\text { g.tec } \\
\text { USBamp } \\
\text { (7-ch) }\end{array}$ & $\begin{array}{l}0 \text { to } 2 \text {-back } \\
\text { letter task }\end{array}$ & $\begin{array}{l}\text { SVM } \\
\text { (binary) }\end{array}$ & $\begin{array}{l}68 \% \\
(2 \text {-classes }) \\
(0 \text { vs. } 2 \text {-back })\end{array}$ \\
\hline $\begin{array}{l}\text { Wang } \\
\text { et al. [34] }\end{array}$ & $\begin{array}{l}\text { Emotiv } \\
\text { EPOC } \\
(14-\mathrm{ch})\end{array}$ & $\begin{array}{l}0 \text { to } 3 \text {-back } \\
\text { (letter, spatial } \\
\text { tasks) }\end{array}$ & $\begin{array}{l}\text { SVM } \\
\text { (binary) }\end{array}$ & $\begin{array}{l}81 \% \\
(2 \text {-classes }) \\
(0 \text { - vs. } 1 / 2 / 3 \text {-back })\end{array}$ \\
\hline $\begin{array}{l}\text { Proposed } \\
\text { method }\end{array}$ & $\begin{array}{l}\text { DIY cap } \\
\text { (8-ch) }\end{array}$ & $\begin{array}{l}\text { EC, EO, } \\
1 \text { to } 3 \text {-back } \\
\text { spatial task }\end{array}$ & $\begin{array}{l}\text { Shallow } \\
\text { ConvNet [31] } \\
\text { (5-classes) }\end{array}$ & $\begin{array}{l}\text { 63\% (5-classes) } \\
>83 \% \text { binary } \\
\text { (rest vs. } 1 / 2 / 3 \text {-back) }\end{array}$ \\
\hline
\end{tabular}

\subsection{Results and discussion}

The results are shown in Fig. 3 as confusion matrix and compared with other neuroscience literature in Table 2 . The proposed method exhibits performance matching or exceeding the related works. The resting state (EO, EC) and cognitively engaging state (1/2/3- back) are well separated. EO and EC are also well distinguished. Although there are more misclassifications among $1 / 2 / 3$-back levels, the result agrees with the neuroscience observations.

Overall, we can conclude that the EEG sensing system based on the ADS1299 reference design in the form of a daily garment - a soft cap, achieves similar levels of recognition as other neuroscience studies with dedicated instruments.

\section{FURTHER GARMENT INTEGRATION}

To demonstrate how easily Brainwear can be integrated with other sensing modalities, we expanded the prototype with two more sensing modalities at two sensing nodes (FP1/2) in Fig. 2.

With the Brainwear module, the improved cap is built around a Feather Huzzah32 module with the ESP32 microcontroller. An FSR and a piezoelectric sensor are stacked with the EEG electrode as shown in Fig. 1. A separate quad-channel ADC (TI-ADS1015) drives the FSR and Piezo sensors at $125 \mathrm{~Hz}$ and communicates with the microcontroller with the $\mathrm{I} 2 \mathrm{C}$ bus.

A brief recording of a person wearing the multi-modality cap performing various head movements and facial expressions is shown in Fig. 4. The cap can detect well-known neural processes such as the "Berger effect" [20] that exhibits an increase in the posterior alpha frequency power $(8-12 \mathrm{~Hz})$ when closing the eyes. The figure also shows that the FSR and piezo reveal Ballistocardiogram (BCG) activity on the forehead together with gross motion characteristics such as nodding, shaking the head, yawning, and squeezing the eyes. These results agree with previous studies in which piezoelectric sensors were used to detect BCG and subtle movements or muscle contractions in the face $[5,33]$. The latter study demonstrated how such activity can be decoupled from neural signals employing an adaptive filter. As [36] discovered, such forehead muscle mechanomyography can detect different expressions such as surprise, sadness, anger, among others. In our example, we can also observe distinct characteristics with different expressions, which could offer information on the wearer's facial expression, whereas the EEG data would have been discarded as a motion artifact in neuroscience. 


\section{CONCLUSION AND OUTLOOK}

To conclude, we consider EEG to be a plug-and-play part of a more extensive customizable multi-modal wearable system. We developed an open-source, transparent EEG sensing module that is pin-compatible with the Feather family and protocol-compatible with an even broader scope of embedded processors. A garment prototype validation taken from the standard neuroscience and BCI literature shows that a soft EEG cap achieves performance on par with neuroscience state-of-the-art. Further integration with mechanomyography shows how EEG and other sensing modalities can be integrated into one wearable system, even at the same sensing location, to provide complementary information about the wearer's neural and physiological activities.

We believe our approach will enable more out-of-the-lab research opportunities that combine neural and physical activities, such as cognitive processes during sports activities.

\section{ACKNOWLEDGMENTS}

This work has been supported by the BMBF (German Federal Ministry of Education and Research) in the project HeadSense (number 01IW18001).

\section{REFERENCES}

[1] Alessandra Angelucci, Matteo Cavicchioli, Ilaria A. Cintorrino, Giuseppe Lauricella, Chiara Rossi, Sara Strati, and Andrea Aliverti. 2021. Smart Textiles and Sen sorized Garments for Physiological Monitoring: A Review of Available Solutions and Techniques. Sensors 21, 3 (Jan. 2021), 814. https://doi.org/10.3390/s21030814

[2] Anum Asif, Muhammad Majid, and Syed Muhammad Anwar. 2019. Human stress classification using EEG signals in response to music tracks. Computers in Biology and Medicine 107 (April 2019), 182-196. https://doi.org/10.1016/j.compbiomed. 2019.02.015

[3] Stefano Betti, Raffaele Lova, and Giorgia Acerbi. 2018. Evaluation of an Integrated System of Wearable Physiological Sensors for Stress Monitoring in Working Environments by Using Biological Markers. IEEE Transactions on Biomedical Engineering 65, 8 (Aug. 2018), 1748-1758. https://doi.org/10.1109/TBME.2017. 2764507

[4] Martin G. Bleichner and Stefan Debener. 2017. Concealed, Unobtrusive EarCentered EEG Acquisition: cEEGrids for Transparent EEG. Frontiers in Human Neuroscience 11 (April 2017). https://doi.org/10.3389/fnhum.2017.00163

[5] G Bonmassar. 2002. Motion and Ballistocardiogram Artifact Removal for Interleaved Recording of EEG and EPs during MRI. NeuroImage 16, 4 (Aug. 2002), 1127-1141. https://doi.org/10.1006/nimg.2002.1125

[6] Anne-Marie Brouwer, Maarten A Hogervorst, Jan B F van Erp, Tobias Heffelaar Patrick H Zimmerman, and Robert Oostenveld. 2012. Estimating workload using EEG spectral power and ERPs in the n-back task. Fournal of Neural Engineering 9, 4 (Aug. 2012), 045008. https://doi.org/10.1088/1741-2560/9/4/045008

[7] Manuel Reis Carneiro, Anibal T. de Almeida, and Mahmoud Tavakoli. 2020 Wearable and Comfortable e-Textile Headband for Long-Term Acquisition of Forehead EEG Signals. IEEE Sensors fournal 20, 24 (Dec. 2020), 15107-15116. https://doi.org/10.1109/JSEN.2020.3009629

[8] Andrew R. A. Conway, Michael J. Kane, Michael F. Bunting, D. Zach Hambrick, Oliver Wilhelm, and Randall W. Engle. 2005. Working memory span tasks: A methodological review and user's guide. Psychonomic Bulletin \& Review 12, 5 (Oct. 2005), 769-786. https://doi.org/10.3758/BF03196772

[9] Jesus G. Cruz-Garza, Zachery R. Hernandez, Sargoon Nepaul, Karen K. Bradley, and Jose L. Contreras-Vidal. 2014. Neural decoding of expressive human movement from scalp electroencephalography (EEG). Frontiers in Human Neuroscience 8 (April 2014). https://doi.org/10.3389/fnhum.2014.00188

[10] Amanda Fleury, Milad Alizadeh, Gabriel Stefan, and Tom Chau. 2017. Toward fabric-based EEG access technologies: Seamless knit electrodes for a portable brain-computer interface. In 2017 IEEE Life Sciences Conference (LSC). IEEE, Sydney, NSW, 35-38. https://doi.org/10.1109/LSC.2017.8268137

[11] Jérémy Frey. 2016. Comparison of a consumer grade EEG amplifier with medical grade equipment in BCI applications. (2016), 2.

[12] Ramla Ghali, Sébastien Ouellet, and Claude Frasson. 2015. LewiSpace: an Exploratory Study with a Machine Learning Model in an Educational Game. fournal of Education and Training Studies 4, 1 (Oct. 2015), 192-201. https: //doi.org/10.11114/jets.v4i1.1153
[13] Manuel Graña, Marina Aguilar-Moreno, Javier De Lope Asiain, Ibai Baglietto Araquistain, and Xavier Garmendia. 2020. Improved Activity Recognition Combining Inertial Motion Sensors and Electroencephalogram Signals. International fournal of Neural Systems 30, 10 (Oct. 2020), 2050053. https: //doi.org/10.1142/S0129065720500537

[14] David Grimes, Desney S. Tan, Scott E. Hudson, Pradeep Shenoy, and Rajesh P.N. Rao. 2008. Feasibility and pragmatics of classifying working memory load with an electroencephalograph. In Proceeding of the twenty-sixth annual CHI conference on Human factors in computing systems - CHI '08. ACM Press, Florence, Italy, 835. https://doi.org/10.1145/1357054.1357187

[15] Texas Instruments. 2017. ADS1299, ADS1299-6, ADS1299-6 Datasheet.

[16] Johan Martijn Jansma, Nick F. Ramsey, Richard Coppola, and René S. Kahn. 2000. Specific versus Nonspecific Brain Activity in a Parametric N-Back Task. NeuroImage 12, 6 (Dec. 2000), 688-697. https://doi.org/10.1006/nimg.2000.0645

[17] Xiao Jiang, Gui-Bin Bian, and Zean Tian. 2019. Removal of Artifacts from EEG Signals: A Review. Sensors 19, 5 (Feb. 2019), 987. https://doi.org/10.3390/s19050987

[18] Stamos Katsigiannis and Naeem Ramzan. 2018. DREAMER: A Database for Emotion Recognition Through EEG and ECG Signals From Wireless Low-cost Off-the-Shelf Devices. IEEE Journal of Biomedical and Health Informatics 22, 1 (Jan. 2018), 98-107. https://doi.org/10.1109/JBHI.2017.2688239

[19] GH Klem, HO Lüders, HH Jasper, and C Elger. 1999. The ten-twenty electrode system of the International Federation. The International Federation of Clinical Neurophysiology. 52 (1999), 3-6. http://europepmc.org/abstract/MED/10590970

[20] Michael Thomas Knierim, Christoph Berger, and Pierluigi Reali. 2021. OpenSource Concealed EEG Data Collection for Brain-Computer-Interfaces - RealWorld Neural Observation Through OpenBCI Amplifiers with Around-the-Ear cEEGrid Electrodes. arXiv:2102.00414 [cs] (Jan. 2021). http://arxiv.org/abs/2102. 00414 arXiv: 2102.00414.

[21] Preeti Kumari, Lini Mathew, and Poonam Syal. 2017. Increasing trend of wearables and multimodal interface for human activity monitoring: A review. Biosensors and Bioelectronics 90 (April 2017), 298-307. https://doi.org/10.1016/j.bios.2016.12.001

[22] Payongkit Lakhan, Nannapas Banluesombatkul, Vongsagon Changniam, Ratwade Dhithijaiyratn, Pitshaporn Leelaarporn, Ekkarat Boonchieng, Supanida Hompoonsup, and Theerawit Wilaiprasitporn. 2019. Consumer Grade Brain Sensing for Emotion Recognition. IEEE Sensors fournal 19, 21 (Nov. 2019), 9896-9907. https://doi.org/10.1109/JSEN.2019.2928781 arXiv: 1810.04582.

[23] Alex Lau-Zhu, Michael P.H. Lau, and Gráinne McLoughlin. 2019. Mobile EEG in research on neurodevelopmental disorders: Opportunities and challenges. Developmental Cognitive Neuroscience 36 (April 2019), 100635. https://doi.org/10. 1016/j.dcn.2019.100635

[24] Aqsa Mehreen, Syed Muhammad Anwar, Muhammad Haseeb, Muhammad Majid, and Muhammad Obaid Ullah. 2019. A Hybrid Scheme for Drowsiness Detection Using Wearable Sensors. IEEE Sensors fournal 19, 13 (July 2019), 5119-5126. https://doi.org/10.1109/JSEN.2019.2904222

[25] Adrian M. Owen, Kathryn M. McMillan, Angela R. Laird, and Ed Bullmore. 2005. $\mathrm{N}$-back working memory paradigm: A meta-analysis of normative functional neuroimaging studies. Human Brain Mapping 25, 1 (May 2005), 46-59. https: //doi.org/10.1002/hbm.20131

[26] J Daniel Ragland, Bruce I Turetsky, Ruben C Gur, Faith Gunning-Dixon, Travis Turner, Lee Schroeder, Robin Chan, and Raquel E Gur. 2015. Working Memory for Complex Figures: An fMRI Comparison of Letter and Fractal n-Back Tasks. (2015), 23.

[27] Usman Rashid, Imran Niazi, Nada Signal, and Denise Taylor. 2018. An EEG Experimental Study Evaluating the Performance of Texas Instruments ADS1299. Sensors 18, 11 (Nov. 2018), 3721. https://doi.org/10.3390/s18113721

[28] Neethu Robinson, Wei Jie Chester, and Smitha Kg. 2021. Use of Mobile EEG in Decoding Hand Movement Speed and Position. IEEE Transactions on HumanMachine Systems 51, 2 (April 2021), 120-129. https://doi.org/10.1109/THMS.2021. 3056274

[29] Amirsaleh Salehzadeh, Andre P. Calitz, and Jean Greyling. 2020. Human activity recognition using deep electroencephalography learning. Biomedical Signal Processing and Control 62 (Sept. 2020), 102094. https://doi.org/10.1016/j.bspc. 2020.102094

[30] Phattarapong Sawangjai, Supanida Hompoonsup, Pitshaporn Leelaarporn, Supavit Kongwudhikunakorn, and Theerawit Wilaiprasitporn. 2020. Consumer Grade EEG Measuring Sensors as Research Tools: A Review. IEEE Sensors fournal 20, 8 (April 2020), 3996-4024. https://doi.org/10.1109/JSEN.2019.2962874

[31] Robin Tibor Schirrmeister, Jost Tobias Springenberg, Lukas Dominique Josef Fiederer, Martin Glasstetter, Katharina Eggensperger, Michael Tangermann, Frank Hutter, Wolfram Burgard, and Tonio Ball. 2017. Deep learning with convolutional neural networks for EEG decoding and visualization: Convolutional Neural Networks in EEG Analysis. Human Brain Mapping 38, 11 (Nov. 2017), 5391-5420. https://doi.org/10.1002/hbm.23730

[32] Lin Shu, Tianyuan Xu, and Xiangmin Xu. 2019. Multilayer Sweat-Absorbable Textile Electrode for EEG Measurement in Forehead Site. IEEE Sensors fournal 19, 15 (Aug. 2019), 5995-6005. https://doi.org/10.1109/JSEN.2019.2912667 
[33] Robert Sowah, Ryan Friedman, Abdul R. Ofoli, and Baffour Sarkodie-Mensah 2019. Think to Speak - A Piezoelectric-EEG system for Augmentative and Alternative Communication (AAC) using Recurrent Neural Networks. In 2019 IEEE Industry Applications Society Annual Meeting. IEEE, Baltimore, MD, USA, 1-6. https://doi.org/10.1109/IAS.2019.8912419

[34] Shouyi Wang, Jacek Gwizdka, and W. Art Chaovalitwongse. 2016. Using Wireless EEG Signals to Assess Memory Workload in the \$n\$-Back Task. IEEE Transaction on Human-Machine Systems 46, 3 (June 2016), 424-435. https://doi.org/10.1109/ THMS.2015.2476818

[35] Yali Zheng, Tracy C. H. Wong, Billy H. K. Leung, and Carmen C. Y. Poon. 2016 Unobtrusive and Multimodal Wearable Sensing to Quantify Anxiety. IEEE Sensors
Journal 16, 10 (May 2016), 3689-3696. https://doi.org/10.1109/JSEN.2016.2539383 [36] Bo Zhou, Tandra Ghose, and Paul Lukowicz. 2020. Expressure: Detect Expression Related to Emotional and Cognitive Activities Using Forehead Textile Pressure Mechanomyography. Sensors 20, 3 (Jan. 2020), 730. https://doi.org/10.3390/ s20030730

[37] Bo Zhou and Paul Lukowicz. 2020. Snacap: snacking behavior monitoring with smart fabric mechanomyography on the temporalis. In Proceedings of the 2020 International Symposium on Wearable Computers. ACM, Virtual Event Mexico, 96-100. https://doi.org/10.1145/3410531.3414305 